\title{
THE HYPERSURFACE CROSS RATIO
}

\author{
TH. MOTZKIN
}

Introduction. In my note on $A 5$ curve theorem generalizing the theorem of Carnot, ${ }^{1}$ I introduced the notion of curve cross ratio. This extension of the ordinary cross ratio is the simplest situationally invariant (3.4) case of the generalized or hypersurface cross ratio of $n+1$ pairs of hypersurfaces in $n$-space which is the subject of the following lines. The generalized cross ratio is at the same time a generalization of the resultant of $n+1$ quantics; the connection between cross ratios and resultants occurred to me when reading a paper of P. Humbert. ${ }^{2}$

The properties of the generalized cross ratio, including extensions of some of those of the ordinary cross ratio, will be developed, together with the similar and interdependent properties of an analogous generalization of the intersection of $n$ hypersurfaces to pairs of hypersurfaces, in §3. This section, much of the contents of which is known, is parallel to $\S \S 1$ and 2 on the ordinary resultant and intersection. In each section, after the definition and fundamental properties, the influence of a rational transformation of coordinates and of permutation, variation and linear combination of the hypersurfaces is studied.

1. The resultant. 1.1. Definition. Let $x=\left(x_{0}, \cdots, x_{n}\right)$ be a point in (complex, or algebraically closed) projective $n$-space, $n \geqq 0$, and $a=\left(a_{0}, \cdots, a_{n}\right)$ a system of $n+1$ quantics of positive degrees $\bar{a}_{0}, \cdots, \bar{a}_{n}$ in the variables $x_{0}, \cdots, x_{n}$. Then the resultant $[a]$ is an irreducible polynomial in the coefficients of $a$ with $\left[x_{k}^{d_{k}}\right]=1$, such that $[a]=0$ if, and only if, an $x \neq 0$ with $a(x)=0$ (that is, $a_{0}(x)=0, \cdots$, $a_{n}(x)=0$ exists. The resultant is unique since the conditions of irreducibility and $\left[x_{k}^{d_{k}}\right]=1$ distinguish it from its powers and multiples respectively. ${ }^{3}$

1.2. Degree. $[a]$ is a quantic of degree $\prod_{k} \bar{a}$ in the coefficients of $a_{k}$. Considering $\bar{a}_{k}$ as degree of the coefficients, we can write $[a] \cdot=\prod \bar{a}$.

Received by the editors November 2, 1944.

${ }_{1}^{1}$ Bull. Amer. Math. Soc. vol. 51 (1945) pp. 972-975.

${ }^{2}$ Sur l'orientation des systèmes de droites, Amer. J. Math. vol. 10 (1888) pp. 258281.

${ }^{3}$ Cf. van der Waerden, Moderne Algebra vol. 2, 1931, pp. 18-21. As || is used for the absolute value, [ ] (already generally used in case of the vector product) is preferable for determinants, resultants and intersections. The product of all degrees shall be denoted by $\prod \bar{a}$, and if $\bar{a}_{k}$ is omitted, by $\prod_{k} \bar{a}(=1$ for $n=0$, as all void products including 0 degree in 3.4 and 3.8 ). 
1.3. Multiplication. If one of the given quantics $a_{k}$ is a product $a_{k}=\prod a_{k}^{(j)}$, we have $[a]=\prod_{j}\left[a_{0}, \cdots, a_{k-1}, a_{k}^{(j)}, a_{k+1}, \cdots, a_{n}\right]$, in short $[\cdots, \Pi, \cdots]=\prod[]$, as both sides vanish simultaneously, are of equal degree, and can be simultaneously equal to 1 .

1.4. Special cases. If $a_{k} \equiv 0$, then $[a]=0$.

For $\prod \bar{a}=1,[a]$ is a determinant.

For $n=1,[a]$ is a product of $\prod \bar{a}$ determinants.

Further note the monomial case $\left[\alpha_{k} x_{k}^{d_{k}}\right]=\prod \alpha_{k}{ }^{\Pi_{k} d}$.

1.5. Rational transformation. Let $f_{0}, \cdots, f_{n}$ be quantics of equal degree $\bar{f}>0$, then $y=f(x)$ defines a rational transformation. If, for $x \neq 0$, also $y \neq 0$, that is, if $[f] \neq 0$, the transformation is called regular; in it, to every $y$, there belong $\bar{f}^{n}$ points $x .^{4}$ Hence, the only regular birational transformations are the projective transformations.

If $a(y)=0$ for $x \neq 0$, then either $y \neq 0,[a]=0$, or $y=0,[f]=0$. Hence $[a(f)]$ must be of the form $c[a]^{r}[f]^{s}, \bar{c}=0$ (that is, $c$ is constant). In the monomial case $a_{k}=\alpha_{k} x_{k} \bar{a}_{k}, f_{k}=\beta_{k} x_{k} \bar{f}^{\bar{f}}$ we have $a_{k}(f)=\alpha_{k} \beta_{k}^{\bar{d}_{k}} x_{k} \bar{f}^{\bar{f} a_{k}}$, $[a(f)]=\prod\left(\alpha_{k} \beta_{k}^{\bar{a}_{k}}\right)^{f^{n} \Pi_{k} a}$. We obtain $[a(f)]=[a]^{\bar{f}^{n}}[f]^{\Pi \tilde{a}}$.

1.6. Permutation. If the quantics $a_{k}$ are permuted, then $[a]$ and the new resultant $\left[a^{\prime}\right]$ are irreducible and of equal degree and vanish together, so $\left[a^{\prime}\right]=c[a], \bar{c}=0$. Choosing quantics which are products of linear forms, we see by 1.3 and the laws of determinants that $c=( \pm 1)^{\amalg d}$ for a permutation of sign \pm 1 .

1.7. Variation. If $\bar{a}_{k} \geqq \bar{a}_{j}, j \neq k$, and if $a_{k}$ is replaced by $a_{k}+\lambda a_{j}$, where $\lambda$ is a quantic of degree $\bar{a}_{k}-\bar{a}_{j}$, then $[a]$ and the new resultant $\left[a^{\prime}\right]$ are of equal degree and vanish together, so $\left[a^{\prime}\right]=c[a], \bar{c}=0$. Choosing $\lambda=0$, we see that $c=1$.

1.8. Linear combination. If several quantics $a_{k}$ of equal degree are replaced by linear combinations $\sum l_{j k} a_{k}, \bar{l}_{j k}=0$, then by repeated application of 1.7 and 1.2 the new resultant is $\left[a^{\prime}\right]=[l]^{\Pi_{k} d}[a]$.

If all degrees $\bar{a}_{k}$ are equal, 1.6-1.8 are special cases of 1.5.

2. The intersection. 2.1. Definition. If $a_{1}, \cdots, a_{n}, n \geqq 1$, are fixed quantics of positive degree, so that the intersection $a_{1}=\cdots=a_{n}=0$ of $n$ hypersurfaces consists only of a finite number of points $P_{i}$ (for $n=2$, quantics without a common factor of positive degree), then for $\bar{l}=1,[l, a]=0$ only if some $l\left(P_{i}\right)=0$; hence $[l, a]=\prod l\left(P_{i}\right)$ with appropriate multiplicities of sum $\prod \bar{a}$ and with a constant factor according to which the coordinates of the points $P_{i}$ are determined. The system of these $P_{i}$, whose order may be changed and whose coordinates may be multiplied by constants, with product 1 , will be de-

4 By Bézout's theorem, if the number of points is finite. But for, say, $y_{0} \neq 0$, any variety of positive dimension of points $x$ with $y_{0} f_{k}(x)-y_{k} f_{0}(x)=0$ would have a point in common with $f_{0}(x)=0$, against the assumption of regularity. 
noted by $P=[a]$ (the same symbol as for the resultant, but referring to $n$ quantics only); we consider the intersection $P$ as product of its points and write accordingly $[l, a]=l([a])$.

For a general $a_{0},\left[a_{0}, a\right]$ and $a_{0}([a])$ (that is, $\prod a_{0}\left(P_{i}\right)$ ) are of equal degree and vanish together, so they differ only by a constant factor $c$. Choosing $a_{0}$ as product of linear forms, we see by 1.3 that $c=1$, so $\left[a_{0}, a\right]=a_{0}([a])$ (well known for $n=1$ ).

2.2. Degree. If $a_{k}$ is replaced by $c a_{k}, \bar{c}=0$, then $[a]$ becomes $c^{\Pi_{k} a}[a]$; we may again say that $[a]$ is of degree $\prod_{k} \bar{a}$ in the coeffcients of $a$, and, as in 1.2 , write $[a] \cdot=\prod \bar{a}$, so the degree of an intersection is the number of its points. The degree of a resultant in one of the hypersurfaces is the number of meets of the others.

2.3. Multiplication. By $[l, a]=l([a])$ and 1.3 we see that again $[\cdots, \Pi, \cdots]=\Pi[]$, the latter symbol denoting juxtaposition (union) of the points of the respective intersections.

2.4. Special cases. If $[a]$ is defined, then no $a_{k}$ is equal to 0 .

For $\prod \bar{a}=1,[a]$ consists of $n$ determinants ("vector product").

In the monomial case we have $\left[\alpha_{k} x_{k}^{a_{k}}\right]=\prod \alpha_{k}{ }^{\Pi_{k} a}(1,0, \cdots, 0)$.

2.5. Rational transformation. Assume $[f] \neq 0$, and $[a(f)]$ definable by 2.1. A point $Q_{i}$ belongs to $[a(f)]$ if $f\left(Q_{i}\right)$ belongs to $[a]$, so we may write $[a(f)]=[a]: f$. To obtain the exact degree and factor of $P$ in $f(Q)$, put $l=x_{k}, l(f)=f_{k}$, then, by 2.1 , the formula of 1.5 becomes $f_{k}(Q)=\left(x_{k}(P)\right)^{f^{n}}[f]^{\Pi \vec{a}}=x_{k}\left(P^{f^{n}}\right)[f]^{\Pi \tilde{a}}$, or $f([a]: f)=P^{f^{n}}[f]^{\Pi \tilde{a}} ; f^{n}$ agrees with 1.5 , fourth line.

Rational variety. If $a_{m+1}=\cdots=a_{n}=0$ is a fixed rational variety $f(t), t=\left(t_{0}, \cdots, t_{m}\right)$, of degree $\bar{f}$ such that to different $t$ there belong different points $f(t)$, then the cross ratios $[a]$ and $[a(f)]$ as functions of the coefficients of $a_{0}, \cdots, a_{m}$ vanish together and are of equal degree, which is the number of meets of the hypersurfaces except $a_{k}=0$, so they differ only by a constant depending on $f$ and on the form $c^{\Pi \tilde{a}}$ (product up to $\bar{a}_{m}$ ). A factor can be given to $f$ so that $c=1$.

For $n=1$, that is, a unicursal curve, $[a(f)]$ is a product of determinants.

If $a_{m+1}=\cdots=a_{n}=0$ is composed of several rational varieties, $[a]$ equals the product of the corresponding cross ratios. For $m=0$ this is again the final formula of 2.1 .

2.6. Permutation. For a permutation of sign $\pm 1,2.1$ and 1.6 give $a^{\prime}=\left( \pm P_{i}\right)$.

2.7. Variation. By 2.1 and $1.7,\left[\cdots, a_{k}+\lambda a_{j}, \cdots\right]=[a]$.

2.8. Linear combination. By 2.7 and 2.2, $\left[a^{\prime}\right]=[l]^{\mathrm{H}_{k} \vec{a}}[a]$.

3. Generalized cross ratio and intersection. 3.1. Definition. If 
$a_{0}, \cdots, a_{n}$ are quantics of positive degree, except $a_{k}$, which is a quotient $b_{k}: c_{k}$ of quantics of positive degree, we may, in agreement with 1.3 , define $[a]$ as $\left[a_{0}, \cdots, a_{k-1}, b_{k}, a_{k+1}, \cdots, a_{n}\right]$ $:\left[a_{0}, \cdots, a_{k-1}, c_{k}, a_{k+1}, \cdots, a_{n}\right]$, and $\lambda b_{k}: \lambda c_{k}, \bar{\lambda}>0$, will give the same value. Likewise, if every $a_{k}$ is a homogeneous rational function $b_{k}: c_{k},[a]$ is defined as quotient of products of $2^{n}$ resultants. This "resultant of rational functions" we call also cross ratio of the corresponding $n+1$ pairs of hypersurfaces $b_{k}=0$ and $c_{k}=0.5$ It may be $0, \infty$, or indeterminate. Artificial indetermination, due to the choice of $\lambda$, must be avoided.

The intersection may equally be defined for rational functions, in agreement with 2.3 , by admitting negative multiplicities of points $P_{i}$, or, in case all multiplicities are 0 , a number without point. And the notions of resultant and intersection remain linked by the relation $\left[a_{0}, a\right]=a_{0}([a])$. Here, for $\bar{a}_{0}=\bar{b}_{0}-\bar{c}_{0}=0$, every factor $a_{0}\left(P_{i}\right)$ equals $d_{i}: e_{i}$, if $P_{i}$ is on the curve $e_{i} b_{0}-d_{i} c_{0}=0$ of the pencil $\left(b_{0}, c_{0}\right), d_{i}$ and $e_{i}$ being constants.

3.2. Degree. $[a]$ is of degree $\prod_{k} \bar{a}$ in the coefficients of $b_{k}$, and of degree $-\prod_{k} \bar{a}$ in the coefficients of $c_{k}$; so we can again write $[a]=\prod \bar{a}$. The same statements hold for an intersection $[a]$ of pairs of hypersurfaces.

3.3. Multiplication. Again, for cross ratios and intersections, $[\cdots, \Pi, \cdots]=\prod[]$; and for both, $\left[\cdots, 1: a_{k}, \cdots\right]=1:[a]$.

3.4. Special cases. No $b_{k}$ or $c_{k}$ is allowed to be congruent to 0 .

In the monomial case, the relations of 1.4 and 2.4 subsist.

If $\bar{a}_{k}=0,[a]$ is of degree 0 in the coefficients of all other quantics; if $\bar{a}_{j}=\bar{a}_{k}=0,[a]$ depends only on the situation of the given hypersurfaces. ${ }^{6}$ In this case, by 3.1 , the cross ratio $[a]$ is a product of "cross ratios $a_{k}\left(P_{i}: Q_{i}\right)=a_{k}\left(P_{i}\right): a_{k}\left(Q_{i}\right)$ of a pair of points and a pair of hypersurfaces of equal degree," another situational invariant. ${ }^{7}$ As $b_{k}\left(P_{i}: Q_{i}\right)$ is the ratio of the first and last coefficient of $z$ in $b_{k}\left(z_{0} P_{i}+z_{1} Q_{i}\right)$, that is, the product of the ratios $-z_{1}: z_{0}$ corresponding to the meets of $b_{k}=0$ with the line $P_{i} Q_{i}$, we see that $a_{k}\left(P_{i}: Q_{i}\right)$ equals a product of ordinary cross ratios on $P_{i} Q_{i}$, each of which is determined by a meet of $b_{k}=0$ and a meet of $c_{k}=0$.

For cross ratios and intersections with constant $a_{k},[a]=a_{k}{ }^{\mathbf{H}_{k} a}$, and with constant $a_{j}$ and $a_{k},[a]=1$. Thus, in contrast to 1.1 , the

${ }^{5}$ Or of the virtual hypersurfaces $a_{k}=0$, van der Waerden, Algebraische Geometrie, 1939, p. 179.

${ }^{6}$ If $\bar{b}_{k}>0, \bar{c}_{k}>0$, we can attain $\bar{a}_{k}=0$ by replacing $b_{k}$ and $c_{k}$ by their own powers.

${ }^{7}$ Special cases of which are the ratio in Newton's theorem on algebraic curves, and, passing to the logarithms, the angle, and Laguerre's "orientation." 
resultant of quantics of non-negative degree may be reducible; and it may be equal to 0 even if an $x \neq 0$ with $a(x)=0$ exists, which happens if there are 2 or more constant $a_{k}$, all of them equal to 0 (this case, it is true, has been excluded). Yet, in formal developments, we shall admit either or both $\bar{b}_{k}=0$ and $\bar{c}_{k}=0$, so we may assume $b_{k}$ and $c_{k}$ without a common factor of positive degree.

3.5. Rational transformation. The relations of 1.5 and 2.5 subsist. We see that, for $\prod \bar{a}=0$, the cross ratio and intersection are projective invariants. They are not invariant to dualization, common points occurring without common tangents and vice versa.

For a general (not necessarily regular) rational transformation and $\bar{a}_{0}=0, a_{0}\left(P_{i}\right)$ in 3.1 remains unaltered; thereby the change of the cross ratio and the intersection can be obtained.

Rational variety. The conclusions of 2.5 subsist; hence, for $\prod \bar{a}=0$, $[a]=[a(f)]$. For a unicursal curve $f(t)$ with inhomogeneous $t$, met by $b_{0}=0, c_{0}=0, b_{1}=0, c_{1}=0$ respectively in the systems of points $f\left(\beta_{0}\right), f\left(\gamma_{0}\right), f\left(\beta_{1}\right), f\left(\gamma_{1}\right)$ or, admitting negative multiplicities, by $a_{0}=0$ and $a_{1}=0$ (of degrees 0 ) in $f\left(\alpha_{0}\right)$ and $f\left(\alpha_{1}\right)$, we obtain $[a]=\alpha_{0}-\alpha_{1}$ $=\left(\beta_{0}-\beta_{1}\right)\left(\gamma_{0}-\gamma_{1}\right):\left(\beta_{0}-\gamma_{1}\right)\left(\gamma_{0}-\beta_{1}\right)$ where $\beta_{0}-\beta_{1}$, and so on, denote products of differences. For a line and $\bar{b}_{0} \bar{c}_{0} \bar{b}_{1} \bar{c}_{1}=1$, this is the ordinary cross ratio.

It would be interesting to know whether similar laws hold for nonunicursal curves.

3.6. Permutation. The relations of 1.6 and 2.6 subsist. Interchanging of $b_{k}$ and $c_{k}$ gives, by 3.3 , the reciprocal value (or negative multiplicities). All other permutations may change more than the sign of $[a]$, or the sign of the multiplicities. Thus a cross ratio in $(n-1)$ space, or an intersection in $n$-space, $n>1$, has not $(2 n)$ ! but (generally) $N=(2 n) !: n ! 2^{n}=1 \cdot 3 \cdots(2 n-1)$ values, and with the reciprocals $2 N$ values. Change of sign occurs only if among the $2 n$ hypersurfaces there are $n$ of even and $n$ of odd degree; these must be combined in pairs, which can be done in $n$ ! ways, so in this case, together with the opposites, there are respectively $N+n$ ! or $2 N+2 n$ ! values.

But the $N$ values are not independent, the number of resultants (or intersections) being only $C_{2 n, n}=(2 n) !: n !^{2}$, that is, 6, 20,70, 252, $924,3432,12870,48620, \cdots$ instead of $3,15,105,945,10395$, $135135,2027025,34459425, \cdots$. Moreover, $a$ depends only on the $C_{2 n, n}-1$ ratios of resultants, and for odd $n$ only on the $C_{2 n, n}: 2$ ratios of a resultant of certain hypersurfaces and that of all other hypersurfaces.

The true sequence of numbers of independent values begins 
$2,5,14, \cdots$ as seen below. The quantics will be denoted by $1,2, \cdots, 2 n$. Cross ratios and intersections are denoted as in the following example. If the given $[a]$ is $[2: 7,3: 5,6: 1,4: 8]$, we bring 1 to the first place: $\pm 1:[a]=[1: 6,2: 7,3: 5,4: 8]$. In the second place we see 6 , which is the 5 th number after 1 ; so we begin our symbol by 5 . The remaining numbers 234578 are now ordered cyclically, beginning after 6 , that is, with 7.7 is brought to the next place, and we write $\pm a=[1: 6,7: 2,3: 5,4: 8]$. The figure after 7 , that is, 2 , is the second in cyclic order; our symbol begins therefore 62.3 is already in the proper place, 5 is second among 458 ; hence (622). 8 is not in its place, so $\pm 1:[a]=(622)$.

$n=2$. We have $(1)=[1: 2,3: 4]=[13][24]:[14][23],(2)=[1: 3,4: 2]$, $(3)=[1: 4,2: 3]$. Multiplying, we get $A:(1)(2)(3)=(-1)$ to the power $(\overline{2})(\overline{3})+(\overline{2})(\overline{4})+(\overline{3})(\overline{4})$, hence there are 2 independent values. ${ }^{8}$

$n=3$. Permuting 345 cyclically, we have, as before, $A:(11)(12)(13)$ $= \pm 1.234$ gives $B:(11)(23)(31)= \pm 1.235$ gives $(12)(23)(43)= \pm 1$, which follows also from the two preceding types $A$ and $B$ ("types" means that the first figures of the symbols may be replaced by their cyclic successors). So the 5 values $\left(\gamma_{1}\right)$ determine the others; they are independent, as, for example, (11) contains [145] which occurs in no other $\left(\gamma_{1}\right)$.

$n=4.234$ gives $C:(111)(251)(311)= \pm 1.235$ gives $(123)(221)(451)$ $= \pm 1$, or, by $B$ and $C, D:(221)= \pm(111)(131)(311)(511) .236$ gives (131)(213)(511) $= \pm 1$, or, by $B$ and $C$, again $D .237$ gives (143)(212)(651) $= \pm 1$, or, by $A B C$, again $D$. Finally, 246 gives $(123)(353)(512)= \pm 1$, or, by $A B C$, a relation $E$ containing (341). By $C, D, E$ respectively, all $(\delta 51),(\delta 21),(\delta 41)$ are expressed by the 14 values $(\delta 11),(\delta 31)$. These are independent, as, for example, (131) contains [1567] which occurs in no other $(\delta 31)$ or $(\delta 11)$, and (111) contains [1457] which occurs in no other $(\delta 11)$.

Every relation for $[a]$ and its permutations holds also if one of the quantics is 1 , that is, for $2 n-1$ hypersurfaces. ${ }^{9}$ The converse is easily seen to be true for relations derived by cyclic permutation of 3 quantics, and 1.6, 2.6 and 3.3. Under the same, perhaps void, restriction the same relations hold for the values $[a]$ formed by $2 n-2$

${ }^{8}$ The relation $[1: 2,3: 4]+[1: 3,2: 4]=1$ by which the $2 N=6$ values of the ordinary cross ratio are shown to be functions of one of them-and which is equivalent to the additivity of linear measure-ceases to hold, for example, for squares of linear forms. Likewise, for general $n$, there hold special relations in the linear case, reducing the above sequence to that of squares $1,4,9, \ldots . A, B$, and so on, and the effect of interchanging $b_{k}$ and $c_{k}$, are cases of logarithmic antisymmetry.

${ }^{9} A$ for $n=3$ and the last quantic equal to 1 is the extension of Carnot's theorem given in my paper loc. cit. p. 976. 
of $2 n-1$ given hypersurfaces, the dimension of space having been diminished by 1 .

3.7. Variation. $[a]$ remains unchanged if $b_{k}$ is replaced by $b_{k}+\lambda b_{j} c_{j}$, or $c_{k}$ by $c_{k}+\lambda b_{j} c_{j}$. Hence $[a]$ is determined if, in case $\bar{b}_{k} \geqq \bar{b}_{j}+\bar{c}_{j}$, instead of $b_{k}$, only the intersection of $b_{k}=0$ with $b_{j} c_{j}=0$ (and thereby also $b_{k}$ ) is known. Cf. the end of 3.1, and of 3.5.

In some cases the cross ratio $\left[a_{0}, a\right]$ is quite independent of $a_{0}$ (of a given degree). ${ }^{10} \mathrm{By} 3.1$, this happens if, and only if, all multiplicities of the points $P_{i}$ of $[a]$ are equal to 0 , that is, if every meet of $n$ hypersurfaces, one of each pair, belongs to a further hypersurface, for example, in the linear combination case 3.8.

Already for $n=2$ this is not the only case of constant $[1, a]$. Let $b_{1}, c_{1}, b_{2}, c_{2}-$ no two of which shall have a common factor of positive degree-have $p \geqq 0$ common points, and $b_{1}, c_{1}, b_{2}$ have $\gamma_{2}$ additional common points, and define $\beta_{1}, \gamma_{1}, \beta_{2}$ similarly. Then, of course, $\bar{b}_{1} \bar{c}_{1} \geqq p+\beta_{2}+\gamma_{2}$ and 5 analogous inequalities hold, of which-by the before-said- 4 are equations if, and only if, $[1, a]$ is constant. The 4 equations give $\prod \bar{a}=0$ (as stated before); let $\bar{a}_{1}=0$, so that $\bar{b}_{1}=\bar{c}_{1}$, $\beta_{1}=\gamma_{1}$, and $\gamma_{2}-\beta_{2}=\bar{b}_{1} \bar{a}_{2}$, which may be assumed not less than 0 . $\bar{b}_{1}, \quad \bar{c}_{2}, \quad \bar{a}_{2}, p, \quad \beta_{1}, \beta_{2}$ are connected by (1) $\bar{b}_{1}{ }^{2} \geqq p+2 \beta_{2}+\bar{b}_{1} \bar{a}_{2}, \quad$ (2) $\bar{c}_{2}{ }^{2}+\bar{c}_{2} \bar{a}_{2} \geqq p+2 \beta_{1}$, (3) $\bar{b}_{1} \bar{c}_{2}=p+\beta_{1}+\beta_{2}$. Eliminating $p$, there remains (4) $0, b_{1} \bar{c}_{2}-\bar{a}_{1} \bar{c}_{2}-\bar{c}_{2}{ }^{2}+\beta_{1} \leqq \beta_{2} \leqq \bar{b}_{1} \bar{c}_{2}-\beta_{1}, b_{1}{ }^{2}-\bar{b}_{1} \bar{a}_{2}-\bar{b}_{1} \bar{c}_{2}+\beta_{1}$. Eliminating $\beta_{2}$, we have (5) $0, \bar{b}_{1} \bar{a}_{2}+\bar{b}_{1} \bar{c}_{2}-\bar{b}_{1}{ }^{2} \leqq \beta_{1} \leqq \bar{b}_{1} \bar{c}_{2}, \quad\left(\bar{a}_{2}+\bar{c}_{2}\right) \bar{c}_{2} / 2$ and $\left(\bar{b}_{1}-\bar{c}_{2}\right) \bar{a}_{2} \leqq\left(\bar{b}_{1}-\bar{c}_{2}\right)^{2},{ }^{11}$ that is, either $S 1: \bar{a}_{2} \leqq \bar{b}_{1}-\bar{c}_{2}$, which may be written $\bar{c}_{2} \leqq \bar{b}_{2} \leqq \bar{b}_{1}$, or $S 2: \bar{a}_{2} \leqq \bar{b}_{1} \leqq \bar{c}_{2}$, which may be written $\bar{b}_{2}-\bar{c}_{2} \leqq \bar{b}_{1}$ $\leqq \bar{c}_{2} \leqq \bar{b}_{2}\left(\bar{a}_{2} \leqq \bar{b}_{1}\right.$ follows by eliminating $\beta_{1}$, or from (1), except for $\bar{b}_{1}=\bar{c}_{1}=0$, a linear combination case). In both cases, the inequality (6) $\bar{b}_{1} \bar{a}_{2}+\bar{b}_{1} \bar{c}_{2}-\bar{b}_{1}{ }^{2} \leqq\left(\bar{a}_{1} \bar{c}_{2}+\bar{c}_{2}{ }^{2}\right) / 2$ is automatically fulfilled: for $S 1$ this is obvious, for $S 2$ we write (6) $\left(2 \bar{b}_{1}-\bar{c}_{2}\right) \bar{a}_{2} \leqq \bar{b}_{1}{ }^{2}+\left(\bar{b}_{1}-\bar{c}_{2}\right)^{2}$, the left side, if greater than 0 , being not greater than $\left(2 \bar{b}_{1}-\bar{c}_{2}\right) \bar{b}_{1}$ not greater than the right side.

For given $\bar{b}_{1}, \bar{c}_{2}$, we now may choose $\bar{a}_{2}>0$ according to $S 1$ or $S 2$, or $S 3: \bar{a}_{2}=0$, with $\bar{b}_{1}>\bar{c}_{2}$, or $S 4: \bar{a}_{2}=0, \bar{b}_{1}=\bar{c}_{2}$; then $\beta_{1}$ according to (5), which in cases $S 1, S 3, S 4$ reduces to $0 \leqq \beta_{1} \leqq\left(\bar{a}_{1}+\bar{c}_{2}\right) \bar{c}_{2} / 2$; then $\beta_{2}$ according to (4), which in case $S 4$ reduces to $\beta_{2}=\beta_{1}>0$ (for $\beta_{1}=0$ we have a linear combination case); then $p$ by (3), $b_{2}, \gamma_{2} . S 3$ and $S 4$ are the situationally invariant cases. Arranging by ascending $\max \left(\bar{b}_{1}, \bar{c}_{2}\right)$, the table of "nonlinear-combination-cases" begins

${ }^{10} \mathrm{By} 3.3$ it is necessary and sufficient for this, that it be so for a linear form $a_{0}=1$. By 3.2 there must be $\prod \bar{a}=0$. If the cross ratio is 1 , it is also independent of $\bar{a}_{0}$.

11 Also immediately as (1) $+(2)-2(3)$. 


\begin{tabular}{c|cc|cccccccccccc|}
$S$ & 1 & 2 & 2 & 1 & 1 & 3 & 3 & 2 & 2 & 4 & 4 & 2 & 2 & 2 \\
\hline $\bar{b}_{1}$ & 1 & 1 & 1 & 2 & 2 & 2 & 2 & 2 & 2 & 2 & 2 & 2 & 2 & 2 \\
$\bar{c}_{2}$ & 0 & 1 & 2 & 0 & 0 & 1 & 1 & 1 & 1 & 2 & 2 & 2 & 2 & 2 \\
$\bar{a}_{2}$ & 1 & 1 & 1 & 1 & 2 & 0 & 0 & 1 & 1 & 0 & 0 & 1 & 1 & 2 \\
$\beta_{1}$ & 0 & 1 & 2 & 0 & 0 & 0 & 0 & 0 & 1 & 1 & 2 & 2 & 3 & 4 \\
$\beta_{2}$ & 0 & 0 & 0 & 0 & 0 & 1 & 2 & 0 & 1 & 1 & 2 & 0 & 1 & 0 \\
\hline$p$ & 0 & 0 & 0 & 0 & 0 & 1 & 0 & 2 & 0 & 2 & 0 & 2 & 0 & 0 \\
$\bar{b}_{2}$ & 1 & 2 & 3 & 1 & 2 & 1 & 1 & 2 & 2 & 2 & 2 & 3 & 3 & 4 \\
$\gamma_{2}$ & 1 & 1 & 1 & 2 & 4 & 1 & 2 & 2 & 3 & 1 & 2 & 2 & 3 & 4 \\
\hline & $A$ & $B$ & & $C$ & $D$ & $E$ & $F$ & $G$ & $H$ & $I$ & $J$ & & &
\end{tabular}

All 10 cases $A, \cdots, J$, containing only lines and conics, are realizable. Of the 4 situationally invariant cases $E, F, I, J$, the latter concerns 4 conics in the position described by the theorem :

Of 4 given pairs of points every 3 pairs are on a conic if, and only if, either (1) all 4 pairs are on a conic, or (2) a point and a line harmonic to the 4 pairs exist.

Indeed, let $B_{1}, C_{1}, B_{2}, C_{2}$ be pairs of points, and $B_{1} C_{1} B_{2}$ on a conic $c_{2}$, and so on. Choose $C_{2}=(I, J)$, then $b_{1}, c_{1}, b_{2}$ are circles. If they coincide, we have case (1); otherwise $B_{1}, C_{1}, B_{2}$ are pairs of inverse points for a certain circle $O$. If $O$ is a line, we have case (2); otherwise, in the inversion at $O, c_{2}$ becomes a quartic $c_{2}^{\prime}$, meeting $c_{2}$ at $B_{1}, C_{1}, B_{2}$ and in 4 points of $O$, which is impossible. ${ }^{12}$

$F$ (on a pair of conics intersecting in 4 points $A B C D$ and the pair of lines $A B, C D$ ) is a case of cross ratio 1 , for the cross ratio is the same for all pairs of conics, as it cannot become 0 (or $\infty$ ), and equals 1 for coinciding conics; for two circles, $F$ becomes the theorem of the radical axis. $E$ concerns two conics as in $F$ and the lines $A B, A C$; by a limiting case of $F$, either conic may be replaced by $B C$ and its tangent at $A$, so the fixed cross ratio equals that of $A B, A C$ and the two tangents. $I$ yields a theorem on 4 circles, which by inversion becomes the statement that a variable circle is met by a fixed triangle and its circumcircle in a constant cross ratio.

3.8. Linear combination. 1.8 and 2.8 extend to the case where the unaltered $a_{k}$ are rational functions.

12 The non-existence of $c_{2}$, in this case, is also easily proved algebraically, or by remarking that the directions of its axes would have to be harmonic to any 2 of the 3 lines through $O$ and $B_{1}, C_{1}, B_{2}$. 
If $m+1>0$ quantics $b_{k}$ are linear forms $\beta_{k}$ of $m+1$ quantics $d_{k}$, and if the corresponding $c_{k}$ are also linear forms $\gamma_{k}$ of $d_{k}$, then by repeated application of 1.8 , or 2.8 , we have $[a]=[\alpha]^{\Pi_{k}}{ }^{d}$, where $\alpha_{k}=\beta_{k}: \gamma_{k}$. In particular we mention that (a) two pairs of hypersurfaces belonging to a pencil are met by every line in the same cross ratio (and by any other curve in a power of that cross ratio)-a generalization of a fundamental theorem of projective geometry-and this cross ratio is a projective invariant; (b) $n$ pairs of hyperplanes through a point are cut by every hyperplane in the same cross ratio.

The cross ratio of $n+1$ pairs of hyperplanes, or points, considered as function of one of them, is a quotient of two quantics of degree $2^{n-1}$, and constant on a hypersurface of degree $2^{n-1}$. For $n=2$, the cross ratio of 3 pairs of points, or lines, is 1 if, and only if, they are on a conic, which is the fact underlying the projective generation of conics. For general $n$, the cross ratio of $n+1$ points $a$ and of the fundamental points is 1 if, and only if, the product of the coaxial minors of even degree of $a$ equals that of those of odd degree. For $n=1$, this leads to $m=0$.

UNIVERSITY OF JERUSALEM 